PSS PROCEEDINGS

\title{
Masses and Decay Constants from Relativistic Highly Improved Staggered Quarks in Full Lattice QCD
}

\section{E. Follana*}

Universidad de Zaragoza

E-mail: efollana@unizar.es

(for the HPQCD collaboration)

I give an update on the status of HPQCD's heavy hisq program. We use a highly improved, relativistic staggered quark action to calculate masses and decay constants for systems with heavy quarks.

The XXVIII International Symposium on Lattice Field Theory

June 14-19,2010

Villasimius, Sardinia Italy

* Speaker. 


\section{Introduction}

Lattice QCD is now in the position of making realistic and precise calculations, from first principles, of some non-perturbative quantities of phenomenological interest [1]. These calculations are crucial as a test of QCD itself, and as an ingredient in experimental searches for physics beyond the standard model.

Lattice methods excel in the precision calculation from first principles of the fundamental parameters of QCD, the quark masses and the strong coupling constant. The lattice determinations of such quantities are amongst the most accurate available today. Gold-plated meson masses and decay constants can also be calculated to high precision.

Here I give an update of HPQCD's calculation of $f_{D_{s}}$ and quark masses, and some preliminary results about the extension of this method to mesons with $b$ quarks.

\section{Relativistic Heavy Quarks}

If we want to discretize a heavy quark of mass $m$ on a lattice with lattice spacing $a$, the discretization errors associated with the mass will typically behave as some power of am. To keep these small we therefore want $a m \ll 1$. On the other hand we usually also have light quarks, and to keep finite-volume errors coming from those under control we need $L a \gg m_{\pi}^{-1}$. It is difficult to simultaneously satisfy both requirements, and in the past some form of effective heavy quark action was used to discretize the charm and bottom quarks.

On the other hand there are a number of important advantages in using the same relativistic discretization for both light and heavy quarks. The parameters of an effective theory have to be matched to QCD, a non-trivial task and a source of additional errors in the calculation. The relativistic theory frequently has some symmetries that get lost in the effective theory: for staggered quarks there's a partially conserved axial current, and as a consequence meson decay constants do not renormalize. Using the same action for all quarks also allows us, for example, to get very precise ratios of quark masses. Finally, when using the same action for all quarks, there are very few free parameters in the lattice formulation, and therefore it provides a very stringent test of the lattice methods: once the few free parameters have been fixed, all the other results, through the entire range of quark masses, should agree with experiment.

We use a discretization of the fermion action that we call Highly Improved Staggered Quarks (HISQ). It has excellent properties, such as discretization errors starting at $\mathscr{O}\left(a^{2} \alpha_{s}, a^{4}\right)$, and much reduced taste-changing interactions. We have throughly tested this action, and in particular have shown in [2] that, combined with the wide range of lattice spacings available in MILC's ensembles, it can be used to accurately discretize charm quarks. We are also starting to use it for b quarks, although in this case an extrapolation in the heavy quark mass is still necessary, because the existing lattices do not allow us to calculate directly at the $b$ mass.

\section{Details of the Lattice QCD Computation}

The free parameters we have in our formulation are the lattice spacing $a$ and the quark masses: a pair of degenerate light quarks, $m_{l}$, and the strange, charm and bottom quark masses, $m_{s}, m_{c}$ 
and $m_{b}$. The lattice spacing $a$ is determined indirectly through the short-distance parameter $r_{1}$, related to the heavy quark potential, and determined on each ensemble by MILC. We then use a combination of several methods to obtain an accurate value in physical units for $r_{1}$ [3]. $m_{s}, m_{c}$ and $m_{b}$ are fixed to their physical value through $m_{K}, m_{\eta_{c}}$ and $m_{\eta_{b}}$. We cannot yet simulate at the physical value of the light quark masses, and therefore they are varied over a range and a chiral extrapolation is performed. The experimental input here is the pion mass, $m_{\pi}$.

We emphasize that once our action is fixed as described above, no more free parameters remain: everything else that comes out of the calculation is a non-trivial result.

The ensembles we use were generated by the MILC collaboration using the ASQTAD staggered quark action and include two degenerate light quarks and a strange quark. We use ensembles with a range of lattice spacings from 0.045 to $0.15 \mathrm{fm}$. The light quark masses range from $m_{s} / 10$ to $m_{s} / 2.5$.

\section{Update on $f_{D_{s}}$}

We have updated our previous calculation of $f_{D_{s}}$ [4], we now have results at two finer lattice spacings and have better tuned charm and strange quark masses, as well as using the improved determination of $r_{1}$.

We obtain as a final result $f_{D_{s}}=0.2480(25) \mathrm{GeV}$ [5]. This improves on our previous determination [4]. The rather significant change $(2 \sigma)$ in the physical value of $f_{D_{s}}$ comes in large part from the change in the value of $r_{1}$ as a result of our improved determination [3]. In figure 1 we present a comparison of our result with experiment. The discrepancy with the world average, $0.2573(53) \mathrm{GeV}$ [6], is now $1.6 \sigma$, and therefore there is no significant " $f_{D_{s}}$ puzzle" anymore. This is a combination of both our calculation coming up and the experimental average coming down.

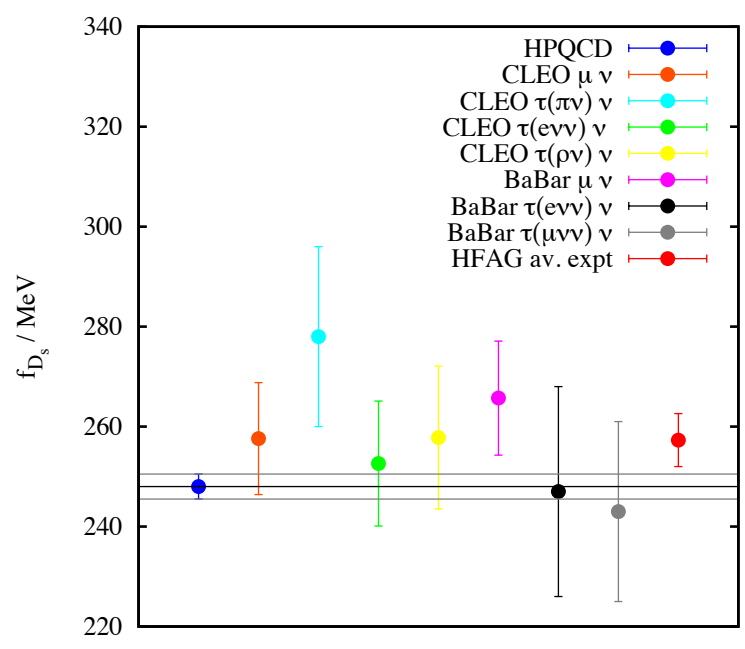

Figure 1: $f_{D_{s}}$ : our calculation compared with experimental results. 


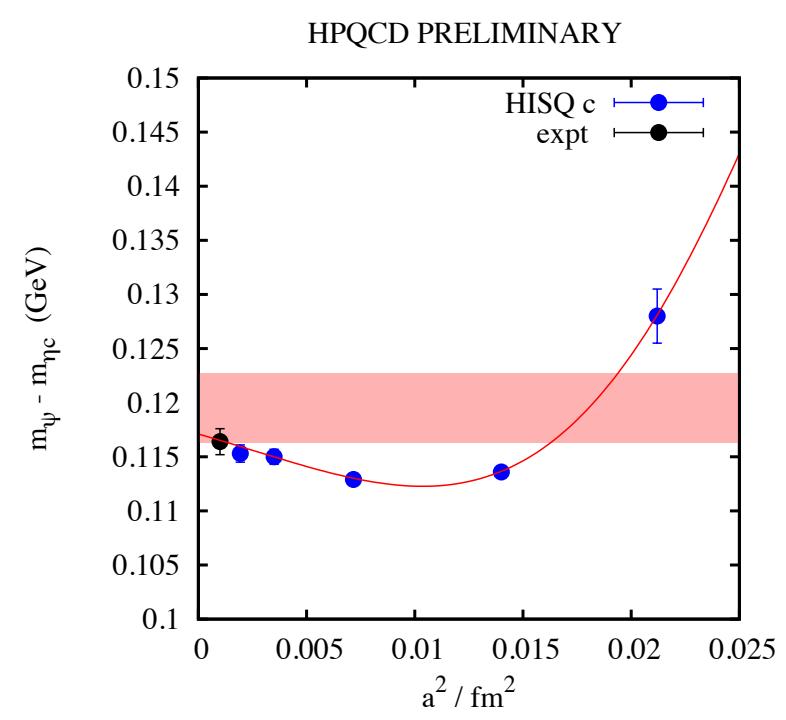

Figure 2: Hyperfine splitting: observe the non-trivial scaling with the lattice spacing.

Having two finer lattice spacings allows us a very good control of discretization errors. As an interesting example of a quantity having quite non-trivial discretization effects, we show in figure 2 the hyperfine splitting in the $c \bar{c}$ system: our preliminary result is $m_{\psi}-m_{\eta_{c}}=118.8(2.4) \mathrm{MeV}$, consistent with the experimental value. We can see the non-monotone behaviour with the lattice spacing $a$, which we can clearly identify only because we have results from the two finest lattices.

\section{Quark masses}

We have previously obtained, using the method of moments and combining high-order continuum perturbation theory with lattice data, a very precise value for the charm quark mass. Our result is $m_{c}\left(3 \mathrm{GeV}, n_{f}=4\right)=0.986(6) \mathrm{GeV}[7,8]$. Because we are using the same relativistic action for all quarks, we can calculate ratios of quark masses very accurately (and completely nonperturbatively); in particular, we can leverage the precision in $m_{c}$ to obtain precise values for strange and light quark masses [9, 8]. We have the result $\frac{m_{c}}{m_{s}}=11.85(16)$ [9], and using our determination of $m_{c}$ gives a strange quark mass $m_{s}\left(2 \mathrm{GeV}, n_{f}=3\right)=92.2(1.3) \mathrm{MeV}$. By using MILC's value for the ratios of light to strange quark masses, we then have $m_{u}\left(2 \mathrm{GeV}, n_{f}=3\right)=2.01(10) \mathrm{MeV}$.

We have also extended the method of moments to the $\mathrm{b}$ quark, and we obtain in this case $m_{b}\left(10(\mathrm{GeV}), n_{f}=5\right)=3.617(25) \mathrm{GeV}$. This is consistent with the nonperturbatively calculated ratio $\frac{m_{b}}{m_{c}}=4.49(4)$.

We show in figure 3 the combined results for the quark masses coming from this analysis, as well as the (much less accurate) current values from the PDG. 


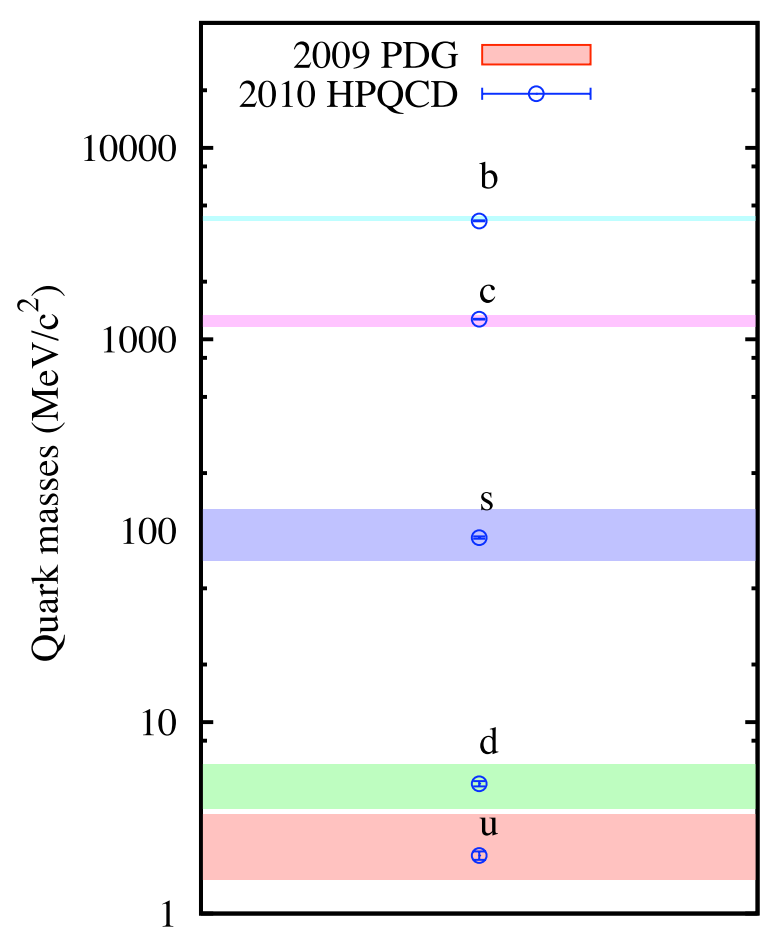

Figure 3: Quark masses: our results compared with the current PDG values.

\section{Towards relativistic b quarks}

We are starting to apply the relativistic formalism to the study of $b$ quarks. Because the lattices we have available now are not fine enough to simulate at the physical value of $m_{b}$, we do calculations at several value of the heavy valence quark mass and we extrapolate to $m_{b}$. We have presented above the results for $m_{b}$. We show in 4 some preliminary results.

\section{Conclusions and Outlook}

The main conclusion is that the use of highly improved relativistic actions and full QCD ensembles with a large range of lattice spacings is at the moment the best method to study systems with a charm quark. We have obtained unprecedentedly accurate results for decay constants $f_{D}$, $f_{D_{s}}$, as well as for meson masses. We also have one of the most accurate determinations of $m_{c}$, and by leveraging the fact that we are using the same action for all quarks, we can cascade that precision to $m_{s}$ and $m_{u, d}$. Another result is a very precise value for $m_{b}$, in this case by extrapolating in the heavy quark mass.

All these results raise the tantalizing prospect of a direct relativistic computation with $\mathrm{b}$ quarks. Although not yet feasible, if we had, for example, an ensemble with a lattice spacing of $0.03 \mathrm{fm}$, we would be able to have at least some result at the physical b mass, therefore decreasing the sizable error coming from the mass extrapolation. This could well be the best way to obtain accurate results in $b$ physics from the lattice. 

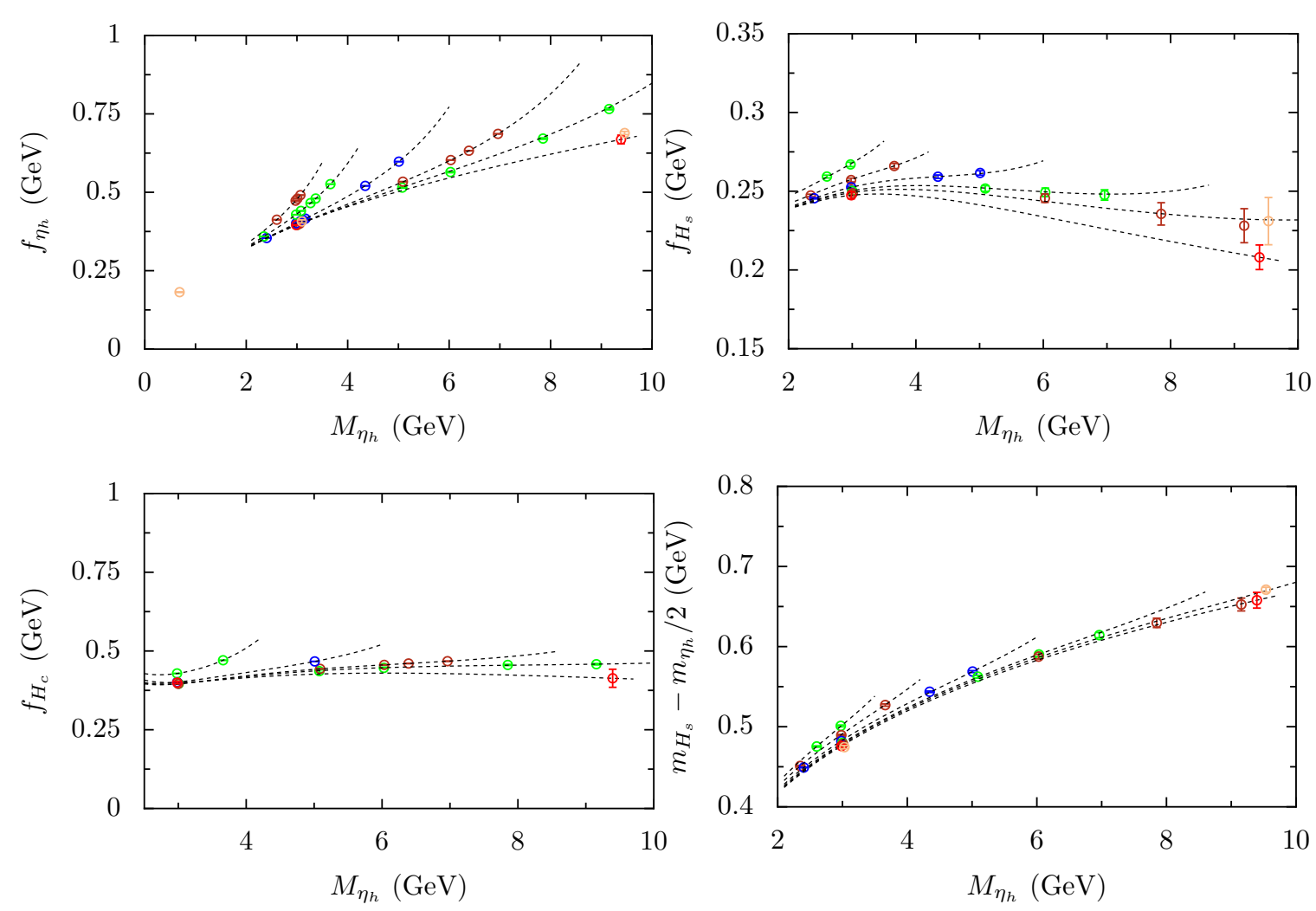

Figure 4: Heavy meson masses and decay constants (preliminary). The different lines correspond to extrapolation in the heavy quark mass for each ensemble. The extrapolation reaches higher quark masses for finer ensembles.

\section{Acknowledgments}

We are grateful to MILC for configurations. Computing was done at the Ohio Supercomputer Center, USQCD's Fermilab cluster and at the Argonne Leadership Computing Facility supported by DOE-AC02-06CH11357. We used chroma for some analyses [10]. We acknowledge support by the STFC, SUPA, NSF and DoE. E. Follana is supported by Ministerio de Ciencia e Innovación through the Ramón y Cajal program.

\section{References}

[1] C. T. H. Davies et al, Phys.Rev.Lett.92:022001,2004 [arXiv:hep-lat/0304004].

[2] E. Follana et al, Phys.Rev.D75:054502,2007 [arXiv:hep-lat/0610092].

[3] C. T. H. Davies et al, Phys.Rev.D81:034506,2010 [arXiv:0910.1229].

[4] E. Follana et al, Phys.Rev.Lett.100:062002,2008 [arXiv:0706.1726].

[5] C. T. H. Davies et al, arXiv:1008.4018.

[6] Heavy Flavor Averaging Group, http://www.slac.stanford.edu/xorg/hfag/charm/index.html.

[7] I. Allison et al, Phys.Rev.D78:054513,2008 [arXiv:0805.2999]. 
[8] C. McNeile et al, Phys.Rev.D82:034512,2010 [arXiv:1004.4285].

[9] C. T. H. Davies et al, Phys.Rev.Lett.104:132003,2010 [arXiv:0910.3102].

[10] R. G. Edwards and B. Joo [SciDAC Collaboration and LHPC Collaboration and UKQCD Collaboration], Nucl. Phys. Proc. Suppl. 140, 832 (2005) [arXiv:hep-lat/0409003]. 